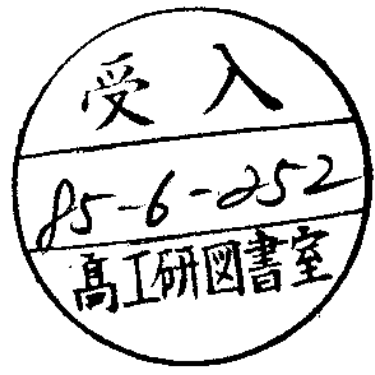

CERN-TH. $4159 / 85$

\title{
SUPERSTRING COMPACTIFICATION AND SUPERSYMMETRY BREAKING
}

E. Cohen, John Ellis, C. Gomez ${ }^{*}$ ) and D.V. Nanopoulos

CERN - Geneva

\section{A B S T R A C T}

We set out conditions which enable gaugino condensation to occur in a compactified supergravity model which may be the low energy limit of a superstring theory. We then argue that the scales of compactification, of supersymmetry breaking and of gauge symmetry breaking can be dynamically determined by radiative corrections in an effective no-scale supergravity model. The resulting low energy sector may avold the cosmological gravitino problen - because the gravitino can decay early enough as not to disturb primordial nucleosynthesis.

*) Dpto. de Fisica Teórica, Universidad de Salamanca, Salamanca, Spain.

CERN-TH. $4159 / 85$

Apri1 1985 
The discovery ${ }^{1)}$ that certain superstring theories ${ }^{2)}$ are anomaly-free and may be finite has rekindled hopes that one of them may be the ultimate theory of everything (TOE), and has stimulated new attempts ${ }^{3)-9)}$ to connect superstring theories to phenomenology. Such attempts involve taking the low energy field theory limit, which is an $\mathrm{N}=1, \mathrm{~d}=10$ supergravity theory with an $0(32)$ or more likely an $E_{8} \times E_{8}$ gauge group ${ }^{10)}$, compactifying the six extra dimenstons and extracting a low energy effective $N=1, d=4$ supergravity theory ${ }^{4}$, which turns out ${ }^{5)}$ to have a "no-scale" $\operatorname{SU}(n, 1) / S U(n) \times U(1)$ structure ${ }^{11)-14)}$. Even if none of the known superstring theories turns out to be the TOE, some features of the above programme will probably survive. For example, we argue later that many forms of compactification ${ }^{4)}$ must lead to the same universal no-scale structure $5(14)$ at low energies. Cruclal outstanding problems in any such scenario are the origin of supersymmetry breaking and determination of its scale, as well as the scales of compactification and of weak gauge symmetry breaking.

Many physicists conjecture that gaugino condensation ${ }^{15}$ ) may trigger supersymmetry breaking, and it has recently been shown ${ }^{8}$ ) how even if this does occur the cosmological constant may remain zero, at least in the absence of perturbative radiative corrections. Our main result in this paper is a characterization of the topological condition necessary for gaugino condensation arising from a twisted four-dimensional gauge field configuration in the compactified ten-dimensional field theory. The condition for bilinear gaugino condensation is non-trivial, and we give examples where known twisted gauge configurations only give higher order condensates. If gaugino condensation does occur in some hidden sector, and supersymmetry breaking is then fed through to the observable sector by higher order corrections, then the scales of supersymmetry and of gauge symmetry breaking can in principle be determined dynamically by radiative corrections, as proposed 12)-14) previously in the context of no-scale supersymmetric grand unified theories. Here the same radiative corrections would simultaneously determine dynamically the scales of compactification, of grand unification and of gaugino condensation:

$$
\mu \sim\left(m_{3 / 2} / M_{P C}\right)^{1 / 12} M_{P C}, m_{x} \sim \mu^{4} / M_{P C}^{3},\left\langle T_{\Gamma} \bar{\chi}^{i} \Gamma_{\alpha \beta \gamma} \chi^{i}\right\rangle \sim \mu^{3}
$$

No-scale models favour ${ }^{14)}$ sparticles weighing several hundred GeV, and the gravitino is likely to be even heavier, and hence to have sufficiently many decay modes $3 / 2 \rightarrow X \tilde{X}$ that $1 t$ can decay before nucleosynthesis thus avoiding any cosmological gravitino problem ${ }^{16), 17)}$. 
First we tackle the question of gaugino condensation, and determine circumstances under which it occurs. We work in the context of $E_{8} \times E_{8}$ ' superstring theory ${ }^{10}$ ), but similar arguments could apply in a larger context. The mechanism proposed in Ref. 8) for the breakdown of supersymmetry with a vanishing cosmological constant involves a bilinear condensation of the gaugino fleld in $E_{8}^{\prime}$. This condensation must occur in the full ten-dimensional theory in order for the mechanism to work. If the mechanism could be understood solely as an effect in the four-dimensional theory, the breaking of $R$-invariance in the four-dimensional Lagrangian would be spontaneous and not explicit. Then the term $\mathrm{CM}_{\mathrm{Pl}}^{3}$ in the superpotential, which is essential ${ }^{8}$ ) for the vanishing of the cosmological constant, would be forbidden since it breaks the R-symmetry explicitly.

To obtain a ten-dimensional condensate $\left\langle\operatorname{Tr}\left(\bar{\chi} \Gamma^{\alpha \beta \gamma} \chi\right)\right\rangle \neq 0$ we need topologically non-trivial gauge configurations (torons ${ }^{18)}$ ) which live in four dimensions. Torons are known to lead to $\langle\bar{q} q\rangle$ condensation in $\mathrm{QCD}^{19}$ ) and to a $\langle\lambda \lambda\rangle$ condensate in four-dimensional supersymmetric Yang-Mills theory ${ }^{20)}$, but the reasoning here is more subtle in several respects.

In four-dimensional SUSY Yang-Mills, the existence of a bilinear gluino condensate can already be inferred from the instanton computation ${ }^{21)}$. Instantons lead to $\langle\lambda \lambda(x) \lambda \lambda(y)\rangle \sim \Lambda^{6}$ where $\Lambda$ is the scale parameter of the theory, and the quadrilinear condensate is independent of $x$ and $y$, as a result of supersymme$\operatorname{try}^{21), 22)}$. As $x$ and $y$ are infinitely separated, clustering can be used ${ }^{22}$ to conclude that we must have $\langle\lambda \lambda\rangle \sim \Lambda^{3}$, as confirmed by the toron computation ${ }^{20)}$. In the ten-dimensional theory, the proliferation of indices makes it difficult to conclude on the basis of a ten-dimensional multilinear condensate that the bilinear condensate forms too. We shall see, however, that torons can generate directly the required $\left\langle\operatorname{Tr}\left(\bar{\chi} \Gamma^{\alpha \beta \gamma} \chi\right)\right\rangle$.

We must allow for the possibility that the group $E_{8}^{\prime}$ is broken by the Hosotani mechanism ${ }^{23}$ ) to a subgroup $Q$, which is in general a direct product $Q=Q_{1} \times Q_{2} \times \ldots \times Q_{n}$. Under $E_{8}^{\prime}$, the gauginos transform as the adjoint representation which has dimension 248, and which happens to be also the fundamental representation of that group. Under the unbroken group Q, the 248 wi11 transform as a direct sum of representations of the form $\left(r_{1}, \ldots, r_{n}\right)$. We can only hope to achieve a condensation $\left\langle\operatorname{Tr}\left(\bar{\chi}_{i} \Gamma^{\alpha \beta \gamma} \chi_{i}\right)\right\rangle \neq 0$ in a factor group $Q_{i}$ for which the only massless mode in the direct sum is the adjoint representation, plus perhaps a bunch of singlets. If other representations $r_{i}$ appear, the situation is rather 
similar to massless four-dimensional SUSY QCD, where the existence of massless matter prevents the condensation of either $\lambda \lambda$ or $\bar{\psi} \psi$. This can be proved using supersymmetry and assuming no pathological behaviour of the condensates ${ }^{22}$, and is inevitable in any non-perturbative computation with topologically non-trivial gauge fields where both gauginos and matter fermions have zero modes: the resulting condensate cannot be clustered into condensates involving only one of those species. If, for example, $E_{8}{ }^{\prime}$ is broken to $E_{6} \times S U(3)$, such that both of the factor groups have massless matter in a fundamental representation, then no bilinear gaugino condensate forms. We therefore demand that there exists at least one $Q_{i}$ under which the gaugino $\chi$ is the only massless field transforming non-trivially. In particular, we retain for now the possibility that $Q_{1}$ could be the entire $\mathrm{E}_{8}$ '.

In the presence of a classical background gauge field $A_{\mu}^{i}$ in four dimenstons, belonging only to the gauge group $Q_{i}$, we wish to solve the ten-dimensional Dirac equation

$$
\phi_{10} x=\left(\phi_{6}+\phi_{4}\right) x=0
$$

to find the zero modes $\chi_{0}^{i}$. The four-dimensional covariant derivative contains the gauge connection $A_{\mu}^{i}$ and no spin connection, and the six-dimensional covariant derivative contains only a spin connection. One solution to Eq. (2) is readily found, namely

$$
\chi_{0}^{i}=k \phi^{-3 / 4} F_{\mu \nu}^{i} \Gamma^{\mu \nu} \epsilon
$$

where $\varepsilon$ is the remaining $N=1$ supersymetry on the manifold $M \times K$, $\varepsilon=\sum_{A=1}^{4} \xi_{A} \times \eta_{A}$, where the $\xi_{A}$ are constant spinors operating on four-dimenstonal space-time, and the $\eta_{A}$ are covariantiy constant spinors operating on the internal space. As was shown in Ref. 4), two independent $\eta$ 's obtained with SU(3) holonomy are in fact sufficient. To see that $x_{0}^{i}(3)$ is a zero mode, recall that if $A_{\mu}^{i}$ solves the classical equation of motion, so does its supersymetric partner $\chi^{i 24)}$ so that $\phi_{4} x_{0}^{1}=0$. Then the only dependence of $\chi_{0}^{i}$ on the internal space co-ordinates comes from the $\eta_{A}$ factors in $\varepsilon$ and therefore also $\emptyset_{6} x_{0}^{i}=0$. The constant $\mathrm{k}$ is a normalization factor, which normalizes $\chi_{0}^{i}$ on ten-dimenstonal space-time. Another zero mode is trivially found from $\bar{\chi}_{0}^{i}$. 
In the presence of a gauge configuration with arbitrary Pontryagin number, the solution (3) is not the only one which can be obtained for $\chi^{i}$. In order to get a bilinear condensate we have to make sure that there are no additional zero modes. This can be done when four-dimensional space-time $M$ is compactified to a torus $T^{4}$. Yang-Mills fields on the four-dimensional torus can have fractional Pontryagin number ${ }^{18)}$, and the Atiyah-Singer index theorem will then in some cases guarantee exactly two solutions to $\mathrm{D}_{4} \chi=0$. Any such solution could readily be made into a solution of $D_{10} \chi=0$ using the covariantly constant spinors $\eta_{A}$ as in Eq. (3).

Could the gauge group $Q_{i}$ be the full $E_{8}$ itself? The gauge group $Q_{1}$, when represented in the adjoint representation of the group, forms a real vectorial fibre bundle on $\mathrm{T}^{4}$. The number of gaugino zero modes, according to the AtiyahSinger theorem, will be given by the first Pontryagin number of this bundle. The Pontryagin number can be derived from the Pontryagin number of the associated principal fibre bundle. We find

$$
P_{\text {ad } / P_{\text {prin }}}=C_{2}(\text { ad })
$$

where $\mathrm{C}_{2}$ (ad) is the second Casimir of the adjoint representation, and $P_{\text {ad }}=1 / 16 \pi^{2} \int \operatorname{Tr}\left(F_{\mu \nu} \widetilde{F}^{\mu \nu}\right)_{\text {ad }}$. For $Q=E_{8}^{\prime}$, since $\Pi_{1}\left(E_{8}\right)=0$ the classification of $E_{8}$ vector bundles on $T^{4}$ is the same as that of $E_{8}$ vector bundles on $S^{4}$. The Pontryagin number of principal $\mathrm{E}_{8}$ bundles on $\mathrm{S}^{4}$ is given by the winding number $\Pi_{3}\left(E_{8}\right)=z^{*}$, which implies that the minimal number of gaugino zero modes is $\mathrm{C}_{2}$ (ad) $=60$. The resulting multiple gaugino condensate would contain 60 gaugino fields, which is not exactly what we want and we therefore conclude that $E_{8}{ }^{\prime}$ has to be broken in order to obtain two zero modes.

Could the gauge group $Q_{1}$ be an $S U(N)$ subgroup of $E_{8}$ '? When $S U(N)$ is represented in the adjoint representation, the resulting vector bundle is a $\mathrm{PU}(\mathrm{N})=\mathrm{SU}(\mathrm{N}) / \mathrm{Z}(\mathrm{N})$ bundle. Since $\Pi_{3}(\mathrm{PU}(\mathrm{N}))=\mathrm{Z}$ and since $\mathrm{C}_{2}(\mathrm{ad})=2 \mathrm{~N}$, the regular instanton bundle will have $2 N$ gaugino modes. However, $\Pi_{l}(P U(N))=Z(N)$, and therefore the classification of $\mathrm{PU}(\mathrm{N})$ bundles on $\mathrm{T}^{4}$ is different from that on $\mathrm{S}^{4}$. In fact, we can get non-trivial classical gauge configurations, the torons ${ }^{18}$ ), with $P_{\text {ad }}=2$ (or equivalently $P_{\text {fund }}=1 / \mathrm{N}$ ). These configurations will

\footnotetext{
*) Note that $\Pi_{9}\left(E_{8}\right)=0$ which means that $E_{8}$ has no winding number on $\mathrm{s}^{10}$, and therefore no non-perturbative $\theta$ vacuum angle in the theory before
compactification.
} 
lead to a bilinear gaugino condensate which has a finite limit as the volume of the torus is taken to infinity. The full computation of the condensate is given 1n Ref. 20).

In order to use the toron mechanism, we have seen that we have to break $E_{8}{ }^{\prime}$ to a group containing an $S U(N)$ factor, say

$$
E_{8}^{\prime} \rightarrow \operatorname{SU}(5) \times S U(5), S U(3) \times E_{6}, S U(2) \times E_{7} \text {, ete. }
$$

Under all the possible breakings of $\mathrm{E}_{8}{ }^{\prime}$, the $\underline{248}$ representation splits into representations of the $Q_{i}$ which always include at least one non-trivial matter representation for each of the $Q_{1}$ products. As explained above, these representations have to be disposed of, if a bilinear gaugino condensate is to form. A zero mode of $b_{4}$ will be a zero mode of $D_{10}$ only if it is also a zero mode of $\mathrm{D}_{6}$, namely if it is a zero mode of the internal space. These transform in a characteristic way under the discrete symmetry group $G$, which determines the internal space manifold $\mathrm{K} / \mathrm{G}$. On any manifold with SU(3) holonomy, there is one solution of the type $(0,0)$ and one of the type $\left.\left.(0,3)^{6}\right)^{*}\right)$.

The Hosotani mechanism ${ }^{23)}$ maps the elements $y \in G$ into $U_{g}^{\prime} \in E_{8}$ 'such that $g \rightarrow U_{g}^{\prime}$ is a homomorphism of $G$ into $\left.E_{8} ;\right)^{6}$. The unbroken subgroup of $E_{8}$ will be that which commutes with all the $U_{g}^{\prime}$. The $U_{g}^{\prime}$ themselves form a discrete subgroup of $E_{8}{ }^{\prime}$, which is denoted by $\bar{G}^{\prime}$. Arguing on the basis of Witten's analysis ${ }^{6}$, the only representations of $E_{8}$ ' which survive as zero modes on the manifold $K / G$ are those which are singlets under the diagonal sum $G \oplus \bar{G}^{\prime}$. This enables us to dispose of unwanted zero modes.

Take for example the manifold ${ }^{4)} \mathrm{K}=\mathrm{Y}(4 ; 5)$, the solution of a quintic equation in $\mathrm{CP}^{4}$. The discrete symmetry of the manifold is $G=\mathrm{Z}(5) \times \mathrm{Z}(5)$, and the forms $(0,0)$ and $(0,3)$ are both invariant under $G$. This is the general condition for retaining $N=1$ supersymmetry on $K / G$. Let us now choose $\bar{G}$ ' to be also $\mathrm{Z}(5) \times \mathrm{Z}(5)$. This will induce the breaking $\mathrm{E}_{8}{ }^{\prime} \rightarrow \mathrm{SU}(5) \times \mathrm{SU}(5)$ under which the adjoint of $E_{8}$ ' has the decomposition

$$
248=(\underline{24}, \underline{1})+(\underline{1}, \underline{24})+(\underline{10}, \bar{s})+(\underline{10}, \underline{s})+(\underline{5}, 10)+(\underline{5}, \overline{10})
$$

*) A differential form of the type $(p, q)$ has $p$ holomorphic and $q$ antiholomorphic indices. 
Since the solutions of $b_{6} \chi=0$ on $K$ are invariant under $G$, they must be invariant under $\bar{G}^{\prime}$ in order to remain solutions on $\mathrm{K} / \mathrm{G}$. Obviously, the only surviving representations are $(1,24)$ and $(24,1)$. The forms $(0,0)$ and $(0,3)$ for these representations will combine to give one Majorana-Weyl spinor in ten dimensions, the $\varepsilon$ of Eq. (3). When we introduce a toron in either of the SU(5) factors, this will lead to two gaugino zero modes. The gauge invariant condensate in the ten-dimensional theory is $\left\langle\operatorname{Tr}\left(\bar{\chi} \Gamma_{\alpha \beta \gamma} \chi\right)\right\rangle$, which we now compute.

The ten-dimensional field strength $\mathrm{F}_{\mu \nu}^{(10)}$ to be used in Eq. (3) is obtained from the four-dimensional toron $\mathrm{F}_{\mu \nu}^{(4)}$ via the Weyl rescaling of the metric 25 )

$$
F_{\mu \nu}^{(10)}=e^{3 \sigma} F_{\mu \nu}^{(4)}=e^{3 \sigma} F_{\mu \nu}^{\text {toron }}
$$

The action of the toron is

$$
\begin{aligned}
S^{\text {toron }} & =\int \frac{\phi^{-3 / 4}}{4 g_{10}^{2}} T_{r} F_{\mu \nu}^{(10)} F^{\mu \nu(10)} \sqrt{\operatorname{det} g^{(10)}} d^{10} x \\
& =\frac{1}{4} \int \phi^{-3 / 4} e^{3 \sigma} F_{\mu \nu}^{(4)} F^{\mu \nu(4)} d^{4} x
\end{aligned}
$$

with the choice $\mathrm{g}^{2} 0 \mathrm{M}_{\mathrm{Pl}}^{6}=1$. For an $\mathrm{SU}(\mathrm{N})$ gauge theory,

$$
S^{\text {toron }}=8 \pi^{2} / 9_{4}^{2} N
$$

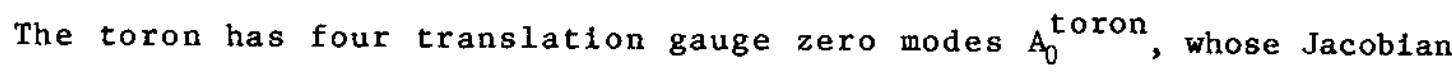
contributes to the condensate a factor [see Ref. 26)]

$$
\begin{gathered}
\frac{d}{g_{10}^{4}} \cdot \mu^{4}\left[\left\{\int\left(A_{0}^{(10)}\right)^{2} \sqrt{\operatorname{det} g^{(0)}} d^{10} x\right\}^{1 / 2}\right]^{4} \\
=d \cdot \mu^{4}\left[\int d^{4} x\left(A_{0}^{\text {toro } x}\right)^{2}\right]^{2}
\end{gathered}
$$

where we have again used the weyl rescaling ${ }^{25)}$, d is a constant of order 1 , and $\mu$ is the scale at which the four-dimensional coupling constant is $g_{4}^{2}=\phi^{3 / 4} \mathrm{e}^{-3 \sigma}$, namely the compactification scale. The two Majorana-Weyl zero modes contribute an additional factor $\mu^{-1}$, and the determinant of non-zero modes is 1 because of supersymetry. We find: 
$\left\langle T_{r} \bar{x}^{i} \Gamma_{\alpha \beta \gamma} X^{i}\right\rangle$
$\sim \mu^{3}\left[\int d^{4} x\left(A_{0}^{\text {toron }}\right)^{2}\right] \int d^{4} z T_{r}\left\langle\bar{x}_{0}^{i} \Gamma_{\alpha \beta \gamma} X_{0}^{i}(x-z)\right\rangle \times e^{-S_{\text {toron }}}$

where $z$ is the "centre" of the toron. The four-dimensional theory sees the plece $\left\langle\operatorname{Tr}\left(\bar{\chi} \Gamma_{i j k} \chi\right)\right\rangle=A \varepsilon_{i j k}$. Note that even though $i, j$ and $k$ are indices on the internal manifold, such a condensate can be realized in terms of the four-dimensional solution $\chi_{0}^{\ddagger}$ of Eq. (3).

Now that we have a gaugino condensate, we indicate how the scales of compactification, of supersymmetry breaking and of weak gauge symmetry breaking may be dynamically determined by radiative corrections. As was shown in Refs. 5) and 8 ), the effective four-dimensional theory at energies below the compactification scale is an $N=1$ supergravity theory containing two gauge singlet chiral superfields

$$
\begin{aligned}
& S \equiv e^{3 \sigma} \phi^{-3 / 4}-i \frac{2 b_{0}}{3} \alpha \equiv S_{R}+i S_{I} \\
& T \equiv e^{\sigma} \phi^{3 / 4}+i a \equiv T_{R}+i T_{I}
\end{aligned}
$$

where $\phi$ is the dilaton in the supergravity multiplet, $\sigma$ determines the scale of the six compactified dimensions:

$$
g_{x I}=e^{\sigma} g_{I J}^{0}: \int_{K} d_{x}^{6} \sqrt{\operatorname{detg}^{0}}=M_{P C}^{-6}
$$

$\alpha$ is the phase of the gaugino condensate, and $a$ is a component of the second rank tensor field, as well as gauge non-singlet chiral superfields denoted generically by $\phi^{i}$. Couplings of the chiral superfields are characterized ${ }^{5)}$ by a Käh1er potential

$$
G=-\ln \left(S+S^{+}\right)-3 \ln \left(T+T^{+}-\phi^{i} \phi_{i}^{+}\right)+\ln |W|^{2}
$$

where $W\left(S, \phi^{i}\right)$ is the chiral superpotential, and by a particularly simple form $\left.{ }^{8}\right)$ of the chiral function characterizing gauge kinetic terms: 


$$
\int d^{2} \theta f_{\alpha \beta} W^{\alpha} W^{\beta}+h . c . \quad, \quad f_{\alpha \beta}=\delta_{\alpha \beta} S
$$

As was shown in Ref. 8), condensation in the hidden sector makes the following contribution to the superpotential:

$$
W(s)=M_{P C}^{3}\left(c+h \operatorname{eop} \frac{-3 s}{2 b_{0}}\right)
$$

in the notation of Ref. 8). Because of the "no-scale" $\operatorname{SU}(n, 1) / \mathrm{SU}(\mathrm{n}) \times \mathrm{U}(1)$ form ${ }^{14)}$ of the T- and $\phi^{i}$-dependent terms in $G$ (15), to which we return later, the $S$ superpotential (17) yields a positive semi-definite effective potential

$$
V=\frac{1}{16} M_{P C}^{4} \frac{1}{S_{R} T_{R}^{3}}\left|c+h\left(\frac{3 S_{R}}{b_{0}}+1\right) \exp \frac{-35}{2 b_{0}}\right|^{2}
$$

As long as $c \neq 0$, the potential (18) may be minimized at $\mathrm{v}=0$ by a suitable dynamical determination of $\mathrm{s}$. The phase $\alpha$ is chosen so that the $c$ and $h$ terms in (18) have opposite signs, and then $S_{R}=e^{3 \sigma_{\phi}-3 / 4}$ is fixed so that $S_{R}=0(1)$. The gravitino then acquires a mass

$$
m_{3 / 2}=M_{P L} e^{G / 2} \sim M_{P L}^{3} \frac{|c|}{S_{R}^{1 / 2} T_{R}^{3 / 2}}=O\left(M_{P C}\right) \times e^{-6 \sigma} \times|c|
$$

and the mass of the axion associated with the phase $\alpha$ is comparable. Since $h$ which is computed from the gaugino condensate is of order 1 , c will typically be a small number: $c=0(1) \exp -8 \pi^{2} / \mathrm{g}^{3} \mathrm{~N}$. Note, however, that so far $\mathrm{T}_{R}$ and hence $e^{\sigma}=\left(S_{R} T_{R}\right)^{1 / 4}$ are undetermined.

To determine them we turn to the observable sector $\phi^{i}$. The origin of their "no-scale" structure ${ }^{14)}$ in the Kähler potential (15) is easy to see and likely to be a general feature of models compactified from higher dimensions. A general feature of such models is that one wants the four-dimensional cosmological constant $\Lambda$ to be (almost) zero, and there to exist a considerable number of massless $\left(m<M_{P \ell}\right)$ chiral superfields. These parametrize the continuous degrees of freedom characterizing a family of related compactification manifolds, each of which has $\Lambda=0$ by construction. Since these models have the same vacuum energy, the values of the chiral superfields are undetermined at this level and remain to be determined by radiative corrections. The only known Kähler potentials which give $\Lambda=0$ automatically for such a continuous range of field 
variables are those containing a $-3 \ell n\left(T+T^{+}-\phi^{1} \phi_{1}^{+}\right)$, 1.e. an $\operatorname{SU}(n, 1) / S U(n) \times U(1)$ structure ${ }^{14)}$, in the context of which just such a scenario for the dynamical determination of light mass scales has been proposed ${ }^{12)-14)}$.

Here, supersymmetry breaking originates from gaugino condensation in the hidden sector, and is absent from the observable sector at tree level, where it can only arise from field-theoretical loops cut-off at the scale of the condensate. It therefore seems quite possible that the observable supersymmetry breaking mass parameters $m_{0}$ (for sleptons) and $\mathrm{m}_{1 / 2}$ (for gauginos) may be somewhat less than $m_{3 / 2}$, though this requires further investigation. Exploiting such a seed of supersymetry breaking, weak gauge symmetry breaking can be triggered in no-scale models ${ }^{14)}$ by a heavy top or heavier quark ${ }^{27)}$, whose large Yukawa coupling renormalizes the Higgs mass squared $\mathrm{m}_{\mathrm{H}}^{2}$ so that although positive (or zero) at the scale of the condensate where $m_{0}$ and $m_{1 / 2}$ are generated, it finally becomes negative at some renormalization scale

$$
\mu_{0}=\mu \exp -\frac{\alpha(1)}{\alpha}
$$

which can easily be $0(100) \mathrm{GeV}$. The scale $\mu_{0}$ is independent of $m_{0}, 1 / 2$ as long as $\mu_{0} \gg m_{0,1 / 2}$. As soon as $m_{H}^{2}<0$ at scales $<\mu_{0}$, weak gauge symmetry breaking is possible with a scale determined by dimensional transmutation ${ }^{28)}$ :

$$
m_{w} \alpha\langle 0|H| 0\rangle=O\left(\mu_{0}\right)
$$

In models such as the present one where the scales $m_{0,1 / 2}$ of global supersymmetry breaking are as yet undetermined, they too can be determined dynamically $^{12)}$ :

$$
m_{0,1 / 2}=\phi\left(\mu_{0}\right) \propto m_{w}
$$

The steps in Eqs. (20) to (22) are famlliar to experts in the field of supergravity model-building: next comes a novelty. By virtue of (22), we now determine through Eq. (19):

$$
T_{R}=O\left(m_{3 / 2}^{-2 / 3}\right) \quad\left(M_{P C} \equiv 1\right)
$$


and hence

$$
e^{\sigma}=O\left(m_{3 / 2}^{-1 / 6}\right)
$$

Thus the scale of compactification is also dynamically determined:

$$
\mu=e^{-\sigma / 2}=O\left(m_{3 / 2}^{1 / 12}\right)
$$

This should come as no surprise, since by having a family of compactification manifolds with $\Lambda=0$ at the tree level one leaves the scale of compactification free to be determined by radiative corrections. Much has been made in the past of the fact ${ }^{29)}$ that perhaps $m_{X} \ll M_{P l}$. Here we find that

$$
m_{x} \sim \mu^{4} /_{P C}^{3} \sim e^{-2 \sigma} \sim O\left(m_{\frac{3}{2}}^{1 / 3}\right)
$$

and is also dynamically determined. This is because, although the scale of the Hosotani symmetry breaking mechanism is $H$, the gauge boson masses that it provides ${ }^{8), 25)}$ are $m_{X}=0\left(\mu^{4} / M_{P \ell}^{3}\right)$. Above this scale, the couplings of all the gauge bosons evolve in an identical unified way.

Finally, we make a cosmological comment. One of the cosmological problems which have bedevilled supersymmetric model-bullders has been the gravitino problem ${ }^{16)}$ : how to avoid gravitino decays from spoiling the successes of conventional primordial nucleosynthesis. Here it is natural to suppose that the gravitino is massive enough to decay before nucleosynthesis, thereby avoiding the problem. We have the suggestion that $m_{3 / 2} \gg m_{0,1 / 2}$, and very possibly $\mathrm{m}_{3 / 2}>\mathrm{m}_{\tilde{q}}, \tilde{l}, \tilde{\mathrm{W}}, \widetilde{B}, \tilde{g}$. The gravitino therefore has many decay modes open to $i t$, and its Iifetime is

$$
T_{3 / 2} \approx \frac{4 \times 10^{8} \mathrm{sec}}{12+2 N_{\phi}} \times\left(\frac{100 \mathrm{GeV}}{m_{3 / 2}}\right)^{3}
$$

where $N_{\phi}$ is the number of light chiral superfields: $N_{\phi}=49$ in the minimal standard model. The gravitino will indeed decay sufficiently rapidiy $\left(\tau_{3 / 2}<100 \mathrm{~s}\right)$ if 


$$
\left(m_{3 / 2} / 100 \mathrm{GeV}\right)>\frac{74}{\left(12+2 N_{\phi}\right)^{1 / 3}}
$$

which is very possibly the case. We conclude that the gravitino problem is potentially solved. 


\section{REFERENCES}

1) M.B. Green and J. Schwarz - Phys.Lett. 149B (1984) 117.

2) For reviews, see:

J. Schwarz - Physics Reports 89 (1982) 223;

M.B. Green - Surv.High Energ.Phys. 3 (1983) 127.

3) E. Witten - Phys.Lett. 149B (1984) 351.

4) P. Candelas, G.T. Horowitz, A. Strominger and E. Witten - Princeton Preprint (December 1984).

5) E. Witten - "Dimensional Reduction of Superstring Mode1s", Princeton Preprint (1985).

6) E. Witten - "Symmetry Breaking Patterns in Superstring Mode1s", Princeton Preprint (1985);

M. Dine, V. Kaplunovsky, M. Mangano, C. Nappi and N. Seiberg - Princeton Preprint (1985).

7) S. Cecotti, J.-P. Derendinger, S. Ferrara, L. Girardello and M. Roncadelli - CERN Preprint TH. 4103 (1985).

8) M. Dine, R. Rohm, N. Seiberg and E. Witten - Princeton Preprint (1985).

9) J.-P. Derendinger, L. Ibañez and H.-P. Nilles - CERN Preprint TH. 4123 (1985).

10) D.J. Gross, A. Harvey, E. Martinec and R. Rohm - Phys.Rev.Lett. 54 (1985) 502 and Princeton Preprint (1985).

11) E. Cremmer, S. Ferrara, C. Kounnas and D.V. Nanopoulos - Phys.Lett. 133B (1983) 61. 12) J. Ellis, A.B. Lahanas, D.V. Nanopoulos and K. Tamvakis - Phys.Lett. 134B
(1984) 429.

13) J. Ellis, C. Kounnas and D.V. Nanopoulos - Nuc1.Phys. B241 (1984) 406.

14) J. Ellis, C. Kounnas and D.V. Nanopoulos - Nuc1.Phys. B247 (1984) 373.

15) H.-P. Nilles - Phys Lett. 115B (1982) 193;

S. Ferrara, L. Girardello and H.-P. Nilles - Phys.Lett. 125B (1983) 457.

16) H. Pagels and J.L. Primack - Phys.Rev.Lett. 48 (1982) 223;

S. Weinberg - Phys.Rev.Lett. 48 (1982) 1303.

17) J. Ellis, A.D. Linde and D.V. Nanopoulos - Phys.Lett. 118B (1982) 59;

J. Ellis, J.E. Kim and D.V. Nanopoulos - Phys.Lett. 145B (1984) 181;

J. Ellis, D.V. Nanopoulos and S. Sarkar - CERN Preprint TH. 4057 (1984).

18) G. 't Hooft - Commun.Math.Phys. 81 (1981) 267;

P. Van Baal - Commun.Math.Phys. 85 (1982) 529.

19) E. Cohen and C. Gomez - Harvard Preprint HUTP-83/A026 (1983). 
20) E. Cohen and C. Gomez - Phys.Rev.Lett. 52 (1984) 237.

21) V.A. Novikov, M.A. Shifman, A.I. Vainshtein and V.I. Zakharov - Nucl.Phys. B229 (1983) 394.

22) G. Veneziano - Phys.Lett. 124B (1983) 357;

D. Amat1, G. Rossi and G. Veneztano - Nuc1.Phys. B249 (1985) 1.

23) X. Hosotani - Phys.Lett. 129B (1983) 193.

24) B. Zumino - Phys.Lett. 69B (1977) 369.

25) A.H. Chamseddine - Nucl.Phys. B185 (1981) 403.

26) G. 't Hooft - Phys.Rev. D14 (1976) 3432.

27) J. E11is, D.V. Nanopoulos and K. Tamvakis - Phys.Lett. 121B (1983) 123; L. Alvarez-Gaumé, J. Polchinski and M.B. Wise - Nuc1.Phys. B221 (1983) 495;

L. Ibañez and C. Lopez - Phys.Lett. 126B (1983) 54.

28) J. Ellis, J.S. Hagelin, D.V. Nanopoulos and K. Tamvakis - Phys.Lett. 125B (1983) 275.

29) S. Dimopoulos, S. Raby and F.A. Wilczek - Phys.Rev. D24 (1981) 1681; M.B. Einhorn and D.R.T. Jones - Nuc1.Phys. B196 (1982) 475;

J. E11is, D.V. Nanopoulos and S. Rudaz - Nuc1.Phys. B202 (1982) 43. 